\title{
LÍMITES DE LA ASISTENCIA MÉDICA Y SOCIAL DURANTE LA GUERRA CIVIL ESPAÑOLA EN UNA CIUDAD DE RETAGUARDIA: LA INCLUSA DEL HOSPITAL PROVINCIAL DE VALENCIA ${ }^{1}$
}

\author{
Xavier García Ferrandis \\ Dr. en Medicina y Cirugía, Universidad Católica de Valencia «San Vicente Mártir» \\ xavier.garcia@ucv.es \\ Àlvar Martínez Vidal \\ Dr. en Medicina y Cirugía, Instituto de Historia de la Medicina y de la Ciencia López Piñero, Universitat de València \\ alvar.martinez@uv.es
}

Recibido: 5 octubre 2015; Aceptado 12 mayo 2016.

Cómo citar este artículo/Citation: García Ferrandis, Xavier y Martínez Vidal, Àlvar (2016), “Límites de la asistencia médica y social durante la Guerra Civil española en una ciudad de retaguardia: la Inclusa del Hospital Provincial de Valencia”, Asclepio, 68 (2): p158. doi: http://dx.doi.org/10.3989/asclepio.2016.30

\begin{abstract}
RESUMEN: Debido a su condición geoestratégica durante la Guerra Civil española, Valencia se convirtió en una de las ciudades republicanas que acogieron mayor número de refugiados, entre ellos muchos niños. En este escenario de crisis demográfica y sanitaria, la ciudad tuvo que reestructurar la red de asistencia con el fin de acoger miles de niños desplazados que, a menudo, tenían a sus familiares en lugares muy lejanos. El artículo analiza la readaptación progresiva que acometió la Inclusa del Hospital Provincial de Valencia. Esta sufrió profundos cambios tanto en su funcionamiento como en su demografía sanitaria, sobre todo como consecuencia del traslado a Valencia de la Inclusa de Madrid. El consiguiente hacinamiento favoreció la degradación de la asistencia sanitaria que allí se dispensaba y la propagación de enfermedades infecciosas entre los niños acogidos.
\end{abstract}

PALABRAS CLAVE: Guerra Civil española; Infancia; Refugiados; Hospital Provincial de Valencia; Inclusa; Madrid.

\section{LIMITS IN MEDICAL CARE AND SOCIAL RELIEF DURING THE SPANISH CIVIL WAR IN A REAR-GUARD CITY: THE INCLUSA OF THE PROVINCIAL HOSPITAL OF VALENCIA}

\begin{abstract}
Due to its geostrategic condition during the Spanish Civil War, Valencia became one of the Republican cities welcoming more refugees, including many children. In this scenario of demographic and health crisis, the town had to restructure the assistance network in order to accommodate thousands of displaced children, who often had their relatives in faraway places. The paper focuses in the progressive rehabilitation undertaken by the Inclusa of Valencia, which was the provincial foundling hospital. This included deep changes both in its operation and in the admission criteria, particularly following the transfer of the Inclusa of Madrid to Valencia. The resulting overcrowding encouraged the degradation of healthcare and the spread of infectious diseases among the welcomed children.
\end{abstract}

KEY WORDS: Spanish Civil War; Childhood; Refugees; Provincial Hospital of Valencia; Foundling hospital; Madrid.

Copyright: (C) 2016 CSIC. Este es un artículo de acceso abierto distribuido bajo los términos de la licencia Creative Commons Attribution (CC BY) España 3.0. 


\section{INTRODUCCIÓN}

En tiempos de guerra, la infancia constituye uno de los colectivos más vulnerables. La crisis sanitaria y social provocada por las penurias que la población civil padece en la retaguardia, principalmente debido a la desprotección y a la carestía de alimentos, resulta devastadora entre los niños. Las enfermedades endémicas de la infancia suelen alcanzar entonces proporciones epidémicas, a la par que se manifiestan con crudeza enfermedades carenciales que en tiempos de paz son poco habituales o están del todo ausentes.

En el caso de la Guerra Civil española, a los sufrimientos padecidos por la población civil, se sumó el bombardeo aéreo y naval de las ciudades republicanas. En esta situación, se planteó la evacuación masiva de niños hacia zonas alejadas de los frentes de batalla, lo que a menudo suponía la dispersión del núcleo familiar y, en paralelo, la necesidad de dar acogida institucional a la infancia refugiada. Alicia Alted ha propuesto la clasificación de esta infancia en tres grupos: los niños trasladados al extranjero en expediciones oficiales durante la contienda; los que partieron al exilio con miembros de sus familias tras la caída del frente catalán a finales de enero de 1939; y los hijos de los vencidos que no fueron evacuados durante la guerra ni partieron después al exilio: los denominados «niños del interior» (Alted, 2003, p. 58).

La historiografía sobre los niños evacuados al extranjero en expediciones oficiales durante la Guerra Civil se ha consolidado en las últimas décadas (Alted, 1996; Zafra, Crego y Heredia, 1997; Labajos y Vitoria, 1997; Alonso, 1998; Alted, 2003; Eloy, 2005). El estudio de este grupo se ha visto favorecido gracias al conocimiento del número y de las vicisitudes de los niños que pasaron al exilio. Sin embargo, la historia de los "niños del interior», es decir, de aquellos niños trasladados por sus familiares y las autoridades a las ciudades republicanas de retaguardia durante la guerra, es menos conocida. El motivo de esta laguna historiográfica bien podría ser la dificultad en determinar el número $-\mathrm{y}$ sus respectivas trayectorias - de los niños que sufrieron el exilio interior, ya que el recuento de los mismos es extremadamente difícil a causa de la propia guerra y de la gran cantidad de movimientos en diferentes direcciones de algunos refugiados (Ortega y Silvestre, 2006, p. 9093). La historiografía sobre los «niños del interior» ha tendido a consolidarse durante los últimos años, aunque desde el prisma del destino de los niños tras la guerra bajo un régimen hostil con los «hijos de los rojos» (Vinyes, 2002).
Por otra parte, en Europa y también en España a partir de las últimas décadas del siglo XIX se desarrolló un movimiento a favor de la infancia cuyo objetivo era la reducción de la mortalidad infantil (Ballester y Balaguer, 1995, pp. 177-192; Palacio, 2003; Rodríguez Ocaña, 2003, pp. 27-36; Perdiguero, 2004). La medicina desempeñó un papel fundamental en este proceso, especialmente en el marco de las campañas sanitarias. De hecho, en todos los países industrializados, el origen de la pediatría como especialidad médica estuvo muy vinculado a actividades de carácter educativo, como las campañas higiénicas de carácter preventivo, en especial la lucha contra la mortalidad infantil (Rodríguez Ocaña, 2003 , p. 34). Asimismo, este movimiento de protección de la infancia debe enmarcarse en otro de carácter general que ha sido denominado «transición demográfico-sanitaria».

En el ámbito valenciano estas cuestiones han sido estudiadas por Carmen Barona y Joan Lloret. Así, Barona, sin franquear la barrera de 1936, ha abordado iniciativas desarrolladas en el ámbito de la protección a la infancia en Valencia, como la Escuela Provincial de Puericultura (Barona, 2002, pp. 349-370); por su parte, Lloret ha analizado la Inclusa de Valencia durante el primer tercio del siglo $\mathrm{XX}$, aunque sin incluir tampoco el período bélico (Lloret, 2015, pp. 155-174).

Finalmente, conviene destacar que la historiografía europea ha mostrado interés en la historia de las inclusas (Kertzer, 1999, pp. 589-603; Abreu, 2003, pp. 37-60; Presciutti, 2011, pp. 752-799), si bien la más estudiada ha sido la de Londres (Nicolson y Kerslake, 1972; McClure, 1981; Levene, 2005, pp. 87-97; Sheetz-Nguyen, 2012). Esta atención no parece ajena a que a mediados del siglo XIX decenas de miles de niños eran abandonados anualmente en las inclusas europeas (Pérez Moreda, 2005, p. 9). En el caso español, la historiografía se ha centrado en la Inclusa de Madrid (Vidal y Vidal, 1995; García Sánchez, 1999; Ramírez, 2002, pp. 109-128; Revuelta, 2012, pp. 645-672; Revuelta 2015, pp. 107-130). Seguramente este afán sea debido al intenso movimiento de la Inclusa de la capital, ya que acogió a unos 340.000 expósitos desde el siglo XVI hasta finales del XX (Pérez Moreda, 2005, p. 9).

Por lo que se refiere a la Guerra Civil y a la inmediata posguerra, hay que destacar los estudios acerca del problema del hambre en Madrid (del Cura y Huertas, 2007) y de la ayuda institucional en la zona franquista prestada por la organización falangista Auxilio Social, sobre todo la distribución de alimentos 
en cantinas a mujeres y a niños (Perdiguero y Castejón, 2009). Sin embargo, la asistencia socio-sanitaria a la infancia refugiada en la zona republicana estaría pendiente de un abordaje en profundidad.

El objetivo de este artículo es determinar el impacto que la Guerra Civil española tuvo en la Inclusa del Hospital Provincial de Valencia, una institución que, emplazada junto a la Facultad de Medicina y los servicios de pediatría (policlínica y sala de niños) del hospital, canalizaba la asistencia a la infancia abandonada en Valencia y su provincia. Somos conscientes de que la Inclusa fue solo una parte - quizá no la más importante- de la compleja red de asistencia sanitaria infantil que se articuló en Valencia durante la contienda. Así, sabemos que en la ciudad funcionaron durante la guerra un total de siete "hogares de la infancia». Básicamente, se trataba de los antiguos asilos infantiles gestionados por la Iglesia que habían sido incautados, sufriendo el preceptivo cambio de nombre. Fueron paradigmáticos por su capacidad los institutos de Asistencia Social «Gabriela Mistral» y "Maestro Ripoll» (antiguas casas de Beneficencia y Misericordia). Como excepción, el Socorro Rojo Internacional fundó y gestionó uno de estos refugios en el incautado convento de San José de la Montaña. Además, se crearon varias guarderías infantiles y un sinfín de refugios que acabarían perdiendo su función inicial para convertirse en infraviviendas donde se hacinaban familias enteras con niños. Asimismo, se habilitaron dos hospitales infantiles, uno destinado a la lucha contra el tracoma y otro de carácter dermatológico. El resto de la red sanitaria infantil estaba integrado por instituciones que ya existían antes de la guerra y que tuvieron que readaptarse a la misma. Debemos incluir aquí el Sanatorio Marítimo de la Malva-rosa, que pasó a llamarse Pablo Iglesias y que en marzo de 1937 recibió a los niños evacuados del Sanatorio de San Rafael de Madrid²; el hospital infantil San Juan de Dios, incautado por el PCE y transformado en Hospital Popular de la Malva-rosa ${ }^{3}$; y el Hospital infantil de Santa Ana, que pasó a denominarse Hospital Neurológico (Selfa, 1953, pp. 32-34).

Nuestra hipótesis de partida es que la gran cantidad de población infantil refugiada que recibió Valencia durante la Guerra Civil condicionó en gran parte la dinámica de funcionamiento de la Inclusa. Para contrastar esta hipótesis y alcanzar así el objetivo propuesto hemos utilizado diversas fuentes primarias localizadas en el Archivo de la Diputación Provincial de Valencia (libros de registro de la Inclusa, filiación de huérfanos, correspondencia, reglamentos, etc.).
Así pues, con este trabajo deseamos realizar una primera aproximación a los «niños del interior» $d u$ rante la contienda española, analizando la vertiente socio-sanitaria desde una perspectiva local. Conviene recordar que algunas de las aportaciones bibliográficas más enriquecedoras en los estudios de la Guerra Civil proceden de la historia local debido a la desintegración del Estado republicano y la dispersión del poder en la España leal (Sagués, 2001, p. 286). El caso de Valencia resulta de especial importancia debido a la situación geoestratégica de la ciudad, situación que se describe posteriormente con cierto detalle.

\section{EL DESPLAZAMIENTO FORZOSO DE LA POBLACIÓN INFANTIL EN LA ZONA REPUBLICANA: VALENCIA, CIUDAD DE ACOGIDA}

El desplazamiento forzoso de población no combatiente es una característica común de las guerras contemporáneas. Sin embargo, la problemática de los refugiados en la guerra de España adquirió una gran proporción debido a la evolución militar y política de la contienda. Efectivamente, con cada victoria franquista el territorio de la República iba reduciéndose y, paradójicamente, iba recibiendo mayor número de refugiados. Además, la dispersión del poder en la España republicana provocó la aparición de numerosos focos de autoridad (comités, sindicatos, partidos políticos, etc.), que tuvieron un efecto negativo en la atención a la población evacuada (multiplicidad de competencias, dispersión de recursos, falta de coordinación). La creación del Ministerio de Sanidad y Asistencia Social en noviembre de 1936 tendió a hacer más eficiente la atención al refugiado; sin embargo, las sucesivas derrotas sufridas por el ejército republicano hicieron que el Gobierno se viera desbordado por el problema de las evacuaciones, por lo que numerosos organismos políticos, sindicales y de ayuda humanitaria -españoles e internacionales - tomaron la iniciativa con el fin de suplir las insuficiencias de todo orden que tenían los organismos oficiales (Alted, 2003, p. 51).

Por tanto, pese a que en ambas zonas en conflicto el niño fue el gran afectado, las características de la contienda hicieron que fuera la República la que tuviera que acometer un abordaje en profundidad del complejo tema de los refugiados, especialmente la población infantil (Fernández, 1987, p. 88). Efectivamente, a medida que la guerra fue extendiéndose por España, el Gobierno republicano optó por trasladar a los refugiados a ciudades de retaguardia, como Valencia o Barcelona, siendo el contingente de niños el más numeroso (Rapport sur la mission sanitaire en 
Espagne, 1937). En las colonias escolares o los hogares de acogida de la España republicana, miles de niños esquivaron los horrores de una guerra que les era completamente ajena (Alted, 1996; Alted, 2003; Escrivà y Maestre, 2011; Morell, 2011; Casademont, 2014). Por ejemplo, en noviembre de 1937 el número de colonias situadas en la provincia de Valencia - solo las sostenidas por el Ministerio de Instrucción Pública- ascendía a 37, siendo 2.294 el número de niños que allí se alojaban (Fernández, 1987, p. 113).

Se estima que a finales de 1936 en el conjunto del territorio de la República el número de refugiados superaba el millón de personas (Rapport sur la mission sanitaire en Espagne, 1937), mientras que en agosto de 1938 la cifra ascendía a dos millones (Ortega y Silvestre, 2006, pp. 90-93) y a finales de ese año rondaba los tres (Clavijo, 2003, p. 90). Consciente de la dimensión que podía alcanzar este problema, el Gobierno de la República legisló al respecto desde bien pronto. Así, el 5 de octubre de 1936, Largo Caballero dispuso la creación en Madrid del Comité Nacional de Refugiados, un organismo que se ramificaba en distintos comités provinciales encargados de realizar un recuento de refugiados y de proporcionarles, además de un lugar de acogida, trabajo y asistencia médica. EI Comité Nacional de Refugiados cambió de nombre varias veces a lo largo de la guerra, hasta que en marzo de 1939 fue disuelto.

Entre la población refugiada en Valencia los niños constituían el contingente más numeroso (Barona, 2002, p. 354). El flujo de población civil que huyó de Madrid a partir de noviembre de 1936 estaba formado sobre todo por maestros y niños (Ortega y Silvestre, 2006, p. 91). En la provincia de Valencia la República habilitó 81 colonias escolares que albergaban cerca de 6.500 niños evacuados de Madrid y otras ciudades castellanas que estaban siendo bombardeadas (Crego Navarro, 1989, pp. 209-328). Se calcula que a finales de 1936 unos 100.000 niños habían salido de Madrid (Escrivà y Maestre, 2011, p. 17), y que a finales de 1938 en Cataluña había más de 200.000 niños refugiados (Fernández, 1987, p. 91).

Las zonas de la España republicana receptoras de la población infantil refugiada tuvieron que readaptar la red de asistencia socio-sanitaria. El caso de Cataluña resulta paradigmático por varios motivos: la cercanía de la frontera, que facilitaba una posible huida a Francia; la autonomía de la región, que favoreció el desarrollo de numerosas iniciativas en el ámbito de la asistencia infantil; y la relativa tranquilidad durante gran parte de la guerra, lo que en la práctica posibilitó la implantación de mecanismos de protección a la infancia. Así, en octubre de 1936 se constituyó el Comitè Central d'Ajut als Refugiats de Catalunya, que en febrero de 1937 tenía bajo su control a diez mil niños. Fue la Generalitat de Catalunya a través de varios organismos oficiales quien asumió la mayor parte de la responsabilidad en el ámbito de la protección de la población infantil evacuada y autóctona (Fernández, 1987, p. 91). Solamente en la ciudad de Barcelona, a finales de marzo de 1937, funcionaban al menos diez instituciones dependientes de la Conselleria de Sanitat i Assistència Social, que acogían un total de 2.328 niños refugiados (Clavijo, 2003, pp. 372-373). Otros organismos oficiales que tomaron parte en la atención a la infancia evacuada fueron Assistència Infantil, Ajut Infantil de Reraguarda y Segell Pro Infància (Fernández, 1987, pp. 93-95), sin olvidar las diversas organizaciones humanitarias internacionales que operaban en la zona (Casademont, 2014, pp. 25-28) ${ }^{4}$. Además de cubrir las necesidades sanitarias y nutritivas de los niños evacuados, Cataluña fue también escenario de numerosas colonias infantiles, donde se dio respuesta a las necesidades educativas de esta población (Fernández, 1987, pp. 96128; Casademont, 2014, pp. 31-46). Todos estos esfuerzos no pudieron evitar la aparición de malnutrición y enfermedades carenciales, debido al elevado contingente de población infantil refugiada, tanto autóctona como evacuada. Así, poco tiempo antes de finalizar la guerra, solo en Cataluña había 1.100.000 niños menores de 15 años, de los que 825.000 presentaban serias carencias (Russell, 1938, p. 1419). La dieta de los niños era deficiente en proteínas, grasas, vitaminas y minerales, lo que provocó la aparición de anemias, raquitismo, marasmo y edemas por desnutrición. Así, el déficit de vitamina $D$ era casi universal en Cataluña en niños menores de tres años (Food in republican Spain, 1939, pp. 278-279).

El caso de Madrid resulta especialmente importante por el contingente tan elevado de niños que fueron evacuados, una dramática medida que estaba más que justificada por motivos de seguridad ante el incremento de los bombardeos sobre la ciudad. En el informe que Federica Montseny, ministra de Sanidad y Asistencia Social, leyó en Ginebra ante la Liga de Naciones se apuntaba que a raíz de una incursión aérea enemiga «en el Dispensario de la Gota de Leche, los miembros de los niños han quedado incrustados en la pared». En 1927 la antigua Inclusa había sido trasladada desde la calle Embajadores a la calle O'Donnell, donde además se construyó un colegio y una maternidad. En 1930 todas estas instalaciones quedaron englobadas en el nuevo Instituto Provincial de Puericultura. La prensa madrileña de la época republicana dedicó numerosos elogios a la nueva Inclusa: "Hemos evitado el hacinamiento (...). Hemos 
desdibujado la estampa de hospital o de asilo que tenía la antigua inclusa. Hoy todo es luz, limpieza, higiene» (Espina, 2005, pp. 450-455). Sin embargo, tras los primeros bombardeos de Madrid en agosto de 1936, la situación se hizo progresivamente insostenible: falta de personal, higiene deficiente, hacinamiento de los niños y falta de medicamentos. Todos estos problemas se tradujeron en una elevada tasa de mortalidad entre los niños a causa de la desnutrición, la disentería y el sarampión. Por estos motivos, el director de la Inclusa de Madrid, Enrique Jaso, organizó la evacuación, que tuvo lugar principalmente entre octubre y noviembre de 1936 (Zafra y Arana, 2015, pp. 8-16). Por tren evacuaron a Valencia a unos mil niños, que fueron acogidos en distintos centros sanitarios y sociales de la ciudad (García Sánchez, 1999; Espina, 2005, pp. 511-514). Pese a todo, conviene destacar que otras organizaciones (como la cenetista Pro Infancia Obrera) también tuvieron un papel destacado en la evacuación de niños madrileños, especialmente durante los primeros meses de la contienda (Clavijo, 2003, p. 145).

Además de las particularidades compartidas con el resto del territorio republicano, en el caso de Valencia hay que añadir una peculiaridad local que condicionó todavía más la política de asistencia a los refugiados: la situación geoestratégica de la ciudad. Próxima a Teruel y, por tanto, cercana a uno de los frentes de guerra más activos del conflicto, Valencia se mantuvo en la retaguardia republicana durante toda la Guerra Civil española. La combinación de estos factores la convirtió en un destino preferente para la población civil evacuada, así como también para los heridos de guerra que llegaban desde los frentes de batalla en oleadas más o menos intermitentes. Así, la población de la ciudad se multiplicó, lo que tuvo consecuencias devastadoras sobre el sistema sanitario (García Ferrandis, 2015).

Aunque las características geoestratégicas de Valencia se dieron en otras ciudades republicanas (como Almería o Murcia), la movilidad migratoria interior durante la guerra se concentró en las provincias más pobladas, Madrid y Barcelona y, de una forma creciente, en Valencia (Ortega y Silvestre, 2006, pp. 90-93). En esta ciudad se estableció un servicio de recepción de refugiados en la Estación del Norte, dado que muchos llegaban en tren (Barona, 2002, p. 353). Este servicio constaba de comedor, duchas y, como puede apreciarse en la imagen 1 , de asistencia médica básica.

Imagen 1. El Comité Nacional de Refugiados en Valencia instaló un servicio médico en la Estación del Norte. En el documento manuscrito se lee: "Médico de Guardia de la Maternidad. Ruego dé ingreso a la refugiada [...]: embarazada de 9 meses, con dolores de parto. Valencia-28-Enero-37". Fuente: Servicio médico de la Estación del Norte. 28 enero 1937. I-2.4, caja 14, legajo 50. Archivo de la Diputación Provincial de Valencia (A.D.P.V.)

COMIté nacional DE REFugiados

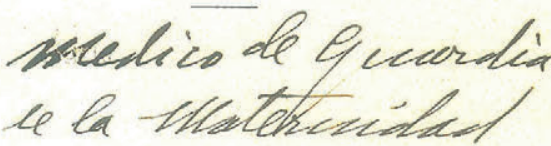
SALVADOR SEGUI, TELÉFONO 19767 VALENCIA

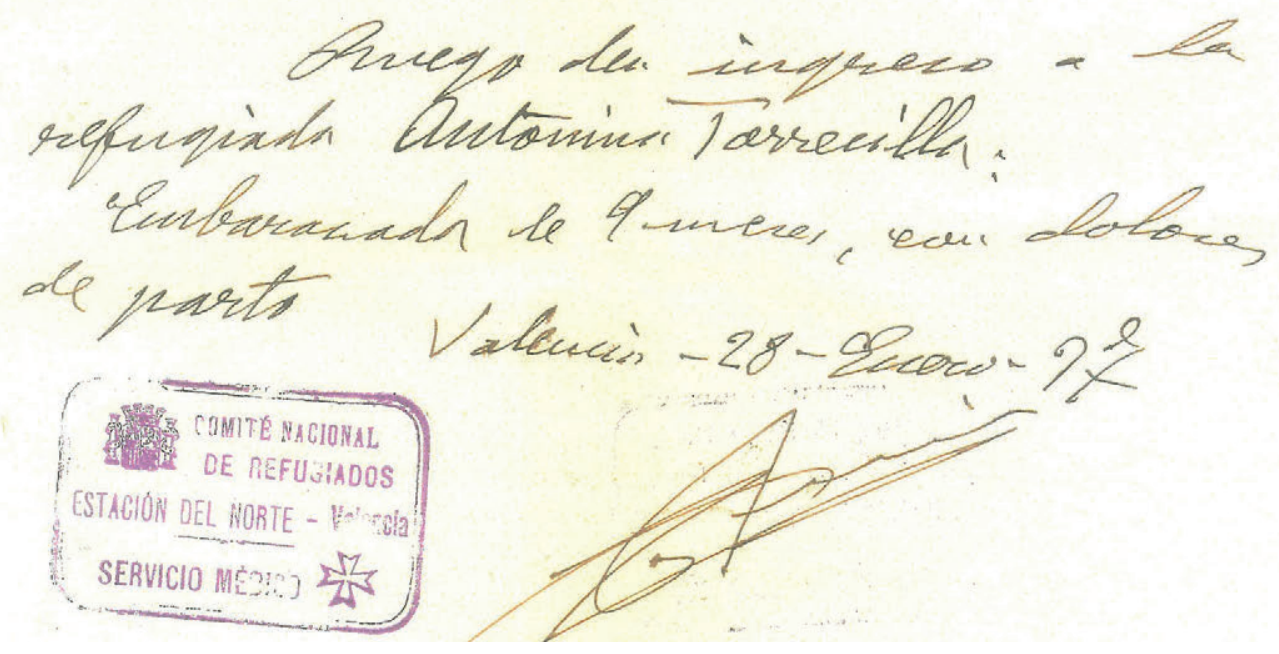




\section{LA INCLUSA DE VALENCIA DURANTE LA GUERRA CIVIL: DEMOGRAFÍA SANITARIA Y DINÁMICA DE FUNCIONAMIENTO}

Ubicada en el recinto del Hospital Provincial, en 1873 la Inclusa de Valencia disponía de una entrada independiente y contaba con dos dormitorios separados por sexos con 40 camas cada uno, y con una sala de mayor tamaño que albergaba unas $130 \mathrm{cu}$ nas. Asimismo, las instalaciones contaban con «dos saloncitos que hacían de enfermerías para cada uno de los sexos» (Barona, 2002, pp. 390-391). El fin primordial de la Inclusa de Valencia era la crianza de los expósitos de la ciudad y su provincia. Tras ingresar, se registraban los datos de filiación y se instalaba al niño en la sala denominada de "prevención» con el fin de someterlo a un reconocimiento médico antes de su traslado a la sección respectiva según su edad y su estado de salud (Barona, 2002, pp. 390-391, 394-395). La alimentación de estos niños, hasta los 20 meses de edad, se realizaba por medio de lactancia, que tenía lugar dentro y fuera de la Inclusa por medio de nodrizas internas y externas. Cuando el niño alcanzaba esta edad, la nodriza externa podía adoptarlo o devolverlo a la Inclusa. Hasta los siete años los niños podían permanecer en la Inclusa; a partir de esa edad eran trasladados a la Casa de Misericordia. Durante el siglo XIX las posibilidades de sobrevivir para los expósitos que permanecían en la Inclusa eran muy bajas, en un momento en que la mortalidad infantil era en sí misma un grave problema de salud pública en la sociedad valenciana. Por este motivo, la política impulsada por los responsables de la Inclusa fue favorecer la salida de los niños para ser criados por nodrizas externas. Con este tipo de crianza el expósito tenía más posibilidades de sobrevivir, ya que era criado en un ambiente familiar y frecuentemente acababa siendo adoptado (Barona, 2002, pp. 421-424).

En 1920 se habilitaron en el Hospital Provincial tres nuevas policlínicas, una de ellas dedicada a la asistencia médica de los niños. Su titular era Ramón Gómez Ferrer (1862-1924), el primer catedrático de pediatría de la Facultad de Medicina de Valencia. Durante la Segunda República los servicios del Hospital Provincial sufrieron una reestructuración para adaptarse a las nuevas especialidades médicas. En este contexto, la Inclusa se incorporó al servicio de «Enfermedades de la infancia» (Barona, 2002, pp. 405, 413). Todo parece indicar, pues, que la proximidad del hospital — sede de la Facultad de Medicina - y la presencia creciente de personal sanitario, así como los cambios demográficos y sanitarios ocurridos desde las últimas décadas del siglo XIX, habrían favorecido la medicalización de la Inclusa.
Como es sabido, en Valencia el desorden derivado del fracaso del golpe de Estado desembocó en la creación del Comité Ejecutivo Popular, un organismo revolucionario integrado por los partidos del Frente Popular y los sindicatos UGT y CNT. Este comité lideró de forma independiente toda la vida política en Valencia y su provincia desde julio de 1936 hasta principios de 1937. Estaba formado por varias delegaciones que asumieron todas las competencias (Propaganda, Guerra, Hacienda, etc.). Para nuestra investigación nos interesan especialmente dos de esas delegaciones: la Delegación de Asistencia Social y la Delegación de Sanidad, también conocida como Comité Sanitario Popular. La reestructuración política iniciada en noviembre de 1936 por el gobierno de Largo Caballero supuso el arrinconamiento progresivo del Comité Ejecutivo Popular de Valencia y sus delegaciones. Las competencias fueron asumidas por el Consejo Provincial de Valencia a través de varias consejerías, entre las cuales cabe resaltar la de Sanidad y la de Asistencia Social (García Ferrandis, 2015, pp. 61-124). En junio de 1938, la Consejería de Sanidad redactaría un protocolo de actuación ante posibles epidemias entre los niños refugiados en Valencia; asimismo, estableció una serie de recomendaciones para su prevención: aseo corporal, baños con jabón e higiene del cabello ${ }^{5}$.

El Comité Nacional de Refugiados, que se hallaba instalado en la calle Salvador Seguí (actual calle Conde de Salvatierra) mientras Valencia ostentaba la capitalidad de la República, consciente de que el problema de los refugiados iba en aumento, se dirigió el 6 de diciembre de 1936 al delegado de Asistencia Social del Comité Ejecutivo Popular en estos términos:

El gran contingente de evacuados que procedente de Madrid llegan a las provincias de Levante, aconseja a este Comité Nacional que se adopten resoluciones rápidas. Esa provincia [Valencia] debe de admitir por ahora un número de refugiados equivalente al $6 \%$ de su población de derecho ${ }^{6}$.

Entre la documentación existente en el Archivo de la Diputación Provincial de Valencia hemos localizado diversas evidencias del movimiento de población infantil hacia Valencia. Así, el 8 de diciembre de 1936, la Delegación de Asistencia Social organizó el traslado a Girona, desde Valencia, de «mil niños de los evacuados del Campo de operaciones» ${ }^{7}$. Por la fecha inferimos que se trataba de niños de la provincia de Teruel, ya que durante diciembre de 1936 las tropas republicanas comenzaron las operaciones para la toma de la ciudad aragonesa ${ }^{8}$. El día de Navidad la Delegación de Asistencia Social del Comité Ejecutivo Popular autori- 
zaba el traslado de «personal indigente evacuado de Madrid entre el que se encuentran numerosos niños» a una colonia instalada en Bétera (Valencia).

Ante esta avalancha, las autoridades valencianas llevaron a cabo diferentes iniciativas para trasladar a niños refugiados fuera de la ciudad de Valencia. La situación empeoró a principios de enero de 1937, cuando la Junta de Defensa de Madrid decretó la evacuación forzosa de la población civil: «van a ser numerosísimas las expediciones de evacuados que se van a formar, algunas de las cuales con destino a esa Capital [Valencia] ${ }^{10}$. Por ejemplo, el 19 de enero de 1937 la Consejería de Sanidad advertía al alcalde de Buñol (Valencia) de la «llegada de ochenta niños más de los procedentes de la evacuación de Madrid a la colonia escolar Juan Marco ${ }^{11}$. Esta colonia era la número 10 en el registro del Ministerio de Instrucción Pública y Sanidad (Escrivà y Maestre, 2011, p. 127).

Por otra parte, a principios de agosto de 1936 el Comité Sanitario Popular solicitó un informe a los jefes de servicio del Hospital Provincial sobre el estado de los departamentos que dirigían. Esta fuente, en su conjunto, revela que el hospital sufría severas deficiencias, especialmente falta de medicamentos, utillaje y personal sanitario. También era evidente la ausencia de una sala de aislamiento para enfermos infecciosos. Por lo que se refiere a la atención pediátrica, el encargado de informar fue el jefe del Servicio de «Enfermedades de la infancia», Jorge Comín Vilar (1890-1956). Médico de la beneficencia provincial, ocupó provisionalmente la plaza de catedrático en 1924, al morir Gómez Ferrer de quien era discípulo, y desde 1931 dirigía la consulta de pediatría del Hospital Provincial. En su informe, Comín señala que en 1936 la Inclusa tenía capacidad para albergar a 30 niños y que durante el primer semestre de ese año el promedio diario de niños había sido de 20. Estas cifras contrastan, a la baja, con las que tenía este establecimiento en 1873, una reducción que se englobaría dentro de la transición demográfica y sanitaria, antes mencionada.

Según Jorge Comín, el personal que atendía la Inclusa valenciana estaba formado por dos médicos y tres enfermeras puericultoras. Tras el examen facultativo, cada ingreso debía ser autorizado por la Dirección del Hospital, quedando a la espera de una pronta adopción. Comín insistía en que los niños albergados en la Inclusa debían ser trasladados fuera tan pronto como su estado lo permitiera, y recordaba que ningún expósito podía ser adoptado antes del cuarto mes de vida, una disposición que como veremos más adelante tendría grandes repercusiones durante la contienda. También advertía de la falta de personal en la Inclusa, reclamando al Comité Sanitario Popular dos médicos y tres enfermeras más para este servicio ${ }^{12}$.

Desde el asedio de Madrid, tal y como hemos señalado anteriormente, gran parte del contingente de población infantil evacuada tenía Valencia como destino. En el Hospital Provincial existía en abril de 1937 un departamento especial denominado «Inclusa de Madrid ${ }^{13}$; de hecho, el hospicio madrileño fue trasladado en su totalidad a Valencia. Los niños madrileños enfermos eran ingresados y, tras su recuperación, eran enviados a una colonia escolar que la Diputación de Madrid había habilitado, tras su confiscación, en un conjunto de residencias de verano de gente adinerada situadas en Navajas ${ }^{14}$, una localidad cercana a Segorbe situada a unos 60 kilómetros de Valencia. Se calcula que en este lugar había unos 400 niños madrileños acogidos en marzo de 1938 (Espina, 2005, pp. 515-516). Todo parece indicar, por tanto, que la Inclusa de Madrid, una vez trasladada a Valencia, dispuso de un "servicio interno» en el Hospital Provincial y de un «servicio externo» en Navajas.

Imagen 2. Oficio de la Junta de Defensa de Madrid relativo a la evacuación de la población civil. Fuente: Sección de Evacuación de la Junta de Defensa de Madrid. 11 enero 1937. D.6.1 caja 20. A.D.P.V.

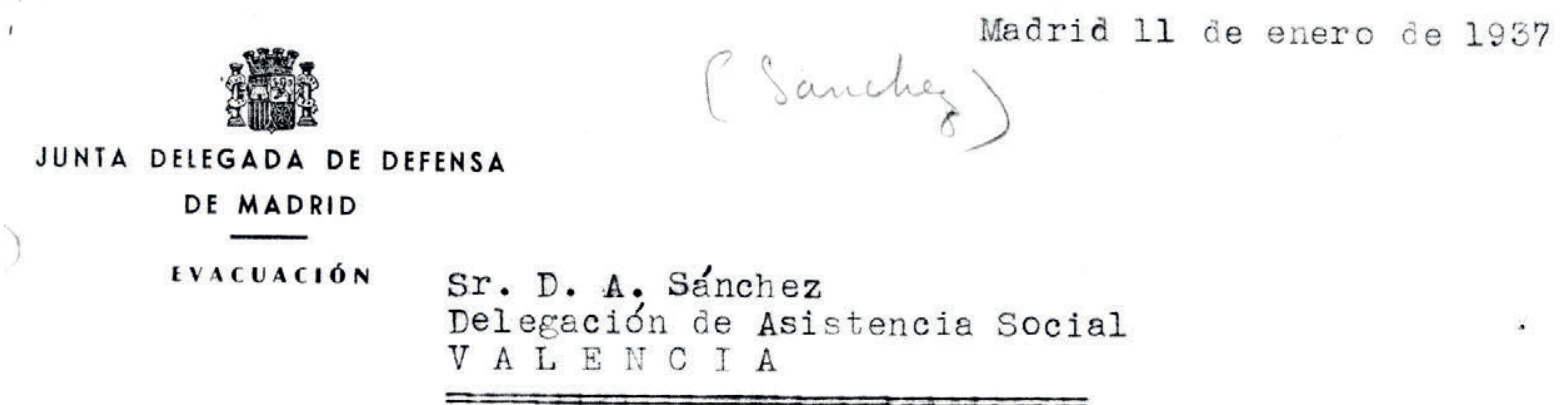


Imagen 3. Oficio de la Diputación Provincial de Madrid referente a sus servicios destacados en Valencia. Fuente: Servicios benéficos en Levante de la Diputación de Madrid. 10 mayo 1937. D.6.1 caja 16. A.D.P.V.

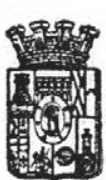

\section{DIPUTACION PROVINCIAL DE MADRID}

\section{SERVICIOS BENEFICOS EN LEVANTE}

La llegada de niños madrileños al Hospital Provincial planteó varios problemas. En primer lugar, la saturación de la dependencias dedicadas a la infancia en el recinto hospitalario valenciano. Así, el 30 de abril de 1937, Jorge Comín denunció ante la Consejería de Sanidad que debido al ingreso de niños madrileños en la Inclusa "quedan actualmente ocupadas las camas destinadas a los destetes procedentes de la Inclusa de Valencia ${ }^{15}$. Pocos días después, el 5 de mayo, tras una nueva denuncia de Comín, la Consejería valenciana de Sanidad se dirigió a la "Inclusa de Madrid en Navajas» en los siguientes términos:

Existen en la Inclusa del Hospital Provincial los niños que proceden de la Inclusa de Madrid y que curados de su enfermedad, no deben permanecer allí, por lo que os ruego dispongáis su traslado ya que su permanencia allí es un peligro y un trastorno para la buena marcha del Establecimiento ${ }^{16}$.

Y, además, no pocos niños evacuados desde Madrid sufrían enfermedades infecciosas altamente contagiosas, lo que alarmaba a las autoridades sanitarias valencianas hasta el punto de exigir al Ministerio de Sanidad una rápida solución a lo que consideraban una amenaza para la salud pública: «Entre los niños de la última expedición de la Inclusa de Madrid hay muchos afectos de conjuntivitis diftérica (...) exigimos resolváis rápidamente para hacer frente a esta epidemia que pudiera acarrear un serio disgusto a la población ${ }^{17}$. Los temores de la Consejería valenciana de Sanidad no eran infundados en modo alguno. De hecho, a partir de 1937 los casos de difteria se incrementaron notablemente en la ciudad entre la población infantil. El citado Jorge Comín firmó a lo largo de un año al menos 52 oficios certificando ingresos de niños aquejados de esta enfermedad en la sala de Pediatría del Hospital Provincial ${ }^{18}$.

Por otro lado, hay que añadir que durante las primeras semanas de la contienda el Comité Sanitario Popular había incautado un internado al Patronato de la Juventud Obrera, que fue habilitado precipitadamente como hospital infantil antitracomatoso. Pese a sus escasos recursos y capacidad, este centro acogió muy pronto a unos 300 niños afectados en su mayoría por esta dolencia oftálmica, pero también por tuberculosis, raquitismo, sarna y otras enfermedades ligadas a las malas condiciones higiénico-sanitarias. Entre los menores ingresados, predominaban los refugiados procedentes de otros lugares de la España republicana (García Ferrandis, 2015, pp. 159-164).

Teniendo en cuenta esta crisis, que era a la vez sanitaria y social, se comprende mejor la dramática situación, próxima al colapso, por la que atravesó la Inclusa valenciana ante la llegada masiva e inesperada de la Inclusa de Madrid. Veamos, pues, el impacto de dicho traslado en la demografía de la Inclusa de Valencia, objeto de este estudio.

El colapso de la Inclusa del Hospital Provincial de Valencia era una realidad ya en diciembre de 1936 debido al gran contingente de población infantil madrileña acogida durante esas fechas. De hecho, el día de Navidad de 1936, el director del hospital se dirigió al Comité Sanitario Popular para solicitar «el envío de 200 juguetes, que no sean de guerra, para los niños de esta Inclusa» ${ }^{19}$. Si recordamos que la capacidad de la Inclusa era de 30 camas, aun aceptando que cada niño pudiera recibir más de un regalo, parece evidente que se había superado ampliamente su capacidad de acogida. Este colapso se tradujo en una degradación de las condiciones higiénicas, que el consejero de Sanidad valenciano denunció afirmando: «he observado que en la Inclusa tal vez por no preocuparse lo suficiente de la ventilación de los locales, se nota un fuerte olor a orines ${ }^{20}$. El hacinamiento también originó la irrupción de enfermedades estacionales infantiles. Así, en el invierno de 1937, los padres de un niño de 18 meses de Alicante decidieron no ingresar a su hijo en la Inclusa por tener noticia de haberse declarado una epidemia de sarampión ${ }^{21}$. Por su parte, Comín en el mes de junio aseguraba que «la época estival y las aglomeraciones de niños por las evacuaciones han determinado este año un aumento de los trastornos digestivos» ${ }^{22}$.

Conviene contextualizar tal declaración por parte de Comín. Efectivamente, al menos desde finales de 1936 se venían registrando, en el Hospital Provincial 
de Valencia, fallecimientos de niños procedentes de la Inclusa de Madrid: solo en diciembre de ese año habían muerto 29 niños madrileños por enfermedades infecciosas, principalmente por difteria y procesos bronconeumónicos, en la sala de Pediatría ${ }^{23}$. La situación de la Inclusa y de esta sala tras un año de guerra debe enmarcarse en un contexto de saturación de todo el Hospital Provincial como consecuencia de la llegada masiva a Valencia de refugiados y heridos de guerra (García Ferrandis, 2011, pp. 364-381).

A continuación veamos el balance de ingresos y salidas en la Inclusa del Hospital Provincial durante la Guerra Civil española. En la gráfica 1 hemos reflejado los ingresos anuales de niños y niñas entre 1934 y 1941 . Se ha ampliado el período de estudio para conocer ambos datos antes y después de la guerra con el objetivo de determinar la influencia de la contienda en los mismos.

Llama la atención el marcado descenso del número de ingresos en la Inclusa valenciana a partir de 1936, que se redujeron a un total de 52 niños entre agosto de 1936 y marzo de 1939. Este descenso no parece corresponderse con lo que se ha señalado anteriormente, que describía una instalación colapsada. De hecho, entre la documentación consultada hemos localizado cuatro cartas fechadas entre abril y octubre de 1937 de familias interesadas en adoptar niños de entre cinco y siete años de la Inclusa. La respuesta de los responsables de la institución es siempre la misma: "Siento tener que manifestarle que no es posible complacerles pues desde hace muchos meses en esta Inclusa [de Valencia] no hay niños y mucho menos de la edad que se precisa» ${ }^{24}$.
¿Es compatible el escenario de una inclusa con signos evidentes de hacinamiento y colapso con una inclusa oficialmente sin niños? Así lo creemos. Nuestra hipótesis es que las instalaciones de la Inclusa del Hospital Provincial estaban abarrotadas por los niños procedentes de la Inclusa de Madrid. Se fundamenta en dos hechos. En primer lugar, en el exiguo, en apariencia, movimiento de niños, que no quedaban anotados en el libro de registro de la Inclusa valenciana y que con toda seguridad contabilizaban en la madrileña. En este contexto encuadramos la siguiente comunicación del director del Hospital a los responsables de Asistencia Social fechada el 25 de octubre de 1937: "Con frecuencia se reciben comunicaciones de ese Departamento interesando ingresos en la Inclusa de menores cuyos antecedentes de filiación se ignoran ${ }^{25}$. A nuestro entender, se trataría de niños de procedencia desconocida cuya presencia no se reflejaba en la documentación oficial, al menos en la de la Inclusa valenciana. También podemos situar en este contexto la queja de Jorge Comín de «que en la Inclusa de Madrid (Departamento especial de este Hospital para dicha Institución) ha ingresado sin mi consentimiento el niño.... ${ }^{26}$. El Consejo Nacional de Tutela de Menores del Ministerio de Justicia (situado en la calle Sorní de la Valencia capitalina) estuvo con frecuencia en el centro de esta controversia al ordenar el ingreso de niños sin contar con el personal de la Inclusa ${ }^{27}$. Asimismo, conviene recordar que, sobrepasado el Gobierno por el problema de las evacuaciones, numerosos organismos políticos, sindicales y de ayuda humanitaria organizaron por su cuenta el socorro a los niños refugiados (Alted, 2003, p. 51). Esta multiplici-

Gráfica 1. Ingresos en la Inclusa de Valencia (1934-1941)

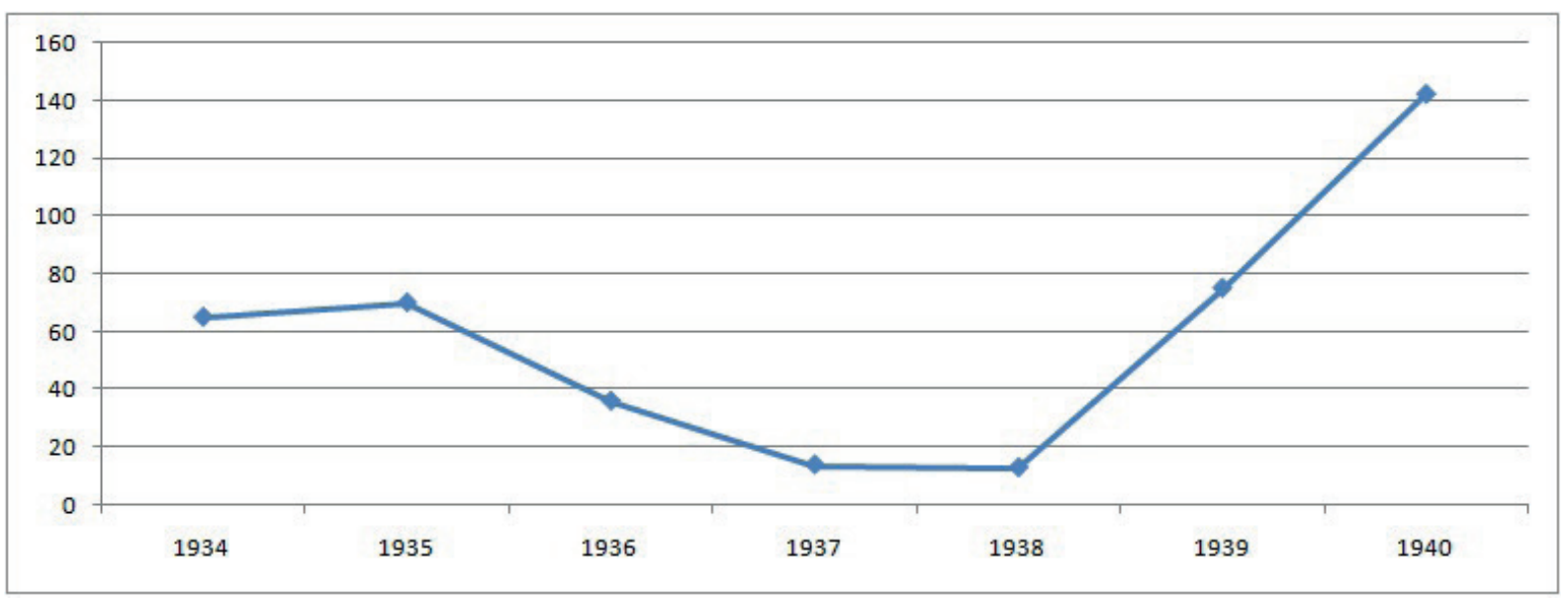

Fuente: elaboración propia a partir de datos de los Libros de Registro de la Inclusa. 1934-1941. A.D.P.V. 
dad de funciones e iniciativas no debió facilitar en absoluto el correcto funcionamiento administrativo de la Inclusa valenciana, cuya capacidad de acogida, más allá de sus límites, se hallaba claramente desbordada.

Nuestra hipótesis se fundamenta en un segundo hecho: la procedencia de los escasos 52 huérfanos que fueron admitidos en la Inclusa entre agosto de 1936 y marzo de $1939^{28}$. Eran todos ellos de muy corta edad: el menor tenía un día y el mayor, 18 meses. El 64\% de la serie analizada era menor de cuatro meses, la edad mínima reglamentaria para ser entregado en adopción.

En la gráfica 2 se observa el origen de estos huérfanos. Como se aprecia, más del $75 \%$ del origen de los ingresos en la Inclusa valenciana durante la guerra estuvo relacionado directamente con el conflicto: hijos de refugiados (33\%), sumados a los niños (36\%) cuyo origen no consta en la documentación consultada por falta de toda referencia. Se trataría de niños de muy corta edad nacidos ocasionalmente en Valencia, fruto de la inestabilidad de la guerra, es decir, hijos de refugiados, a menudo transeúntes y en paradero desconocido. También conviene destacar los hijos de militares (6\%). Entre los numerosos ejemplos localizados resulta paradigmático el caso de una niña, hija de una refugiada procedente de Badajoz, que vivía con su madre en un pueblo de Castellón. La madre murió como consecuencia del vuelco del camión que la evacuaba por segunda vez, y la niña entró en la Inclusa de Valencia el 7 de junio de 1938, unos pocos días antes de la entrada de las tropas franquistas en Castellón. Los combates en esa provincia fueron, sin duda, el motivo que llevó a la malograda madre a iniciar su evacuación a Valencia. El 7 de julio de 1938 esta niña fue reclamada por su padre, soldado del XXI Cuerpo de Ejército republicano ${ }^{29}$. Otro caso parecido lo encontramos en una niña de 11 meses que ingresó en la Inclusa en febrero de 1937: el padre había muerto en el frente de Madrid y la madre había ingresado en el Hospital de Refugiados de Valencia. Estaríamos ante dos posibles evidencias de la persistencia de vínculos entre el niño y su familia biológica.

La documentación consultada apunta hacia la idea de que en la Inclusa del Hospital Provincial durante la Guerra Civil el número de niños autóctonos debió ser escaso, porque se priorizaba el ingreso de los niños evacuados de Madrid y de los hijos de milicianos (locales o forasteros), de militares de paso por Valencia y de refugiados cuyo paradero se desconocía, es decir, niños que en cualquier momento podían ser reclamados por sus padres. La documentación custodiada en el Archivo de la Diputación de Madrid apunta también en esa dirección, ya que revela que numerosos niños evacuados a Valencia a finales de 1936 fueron reclamados por sus padres en los años 1937,1938 y $1939^{30}$.

Gráfica 2. Origen de los huérfanos ingresados en la Inclusa de Valencia (1936-1939)

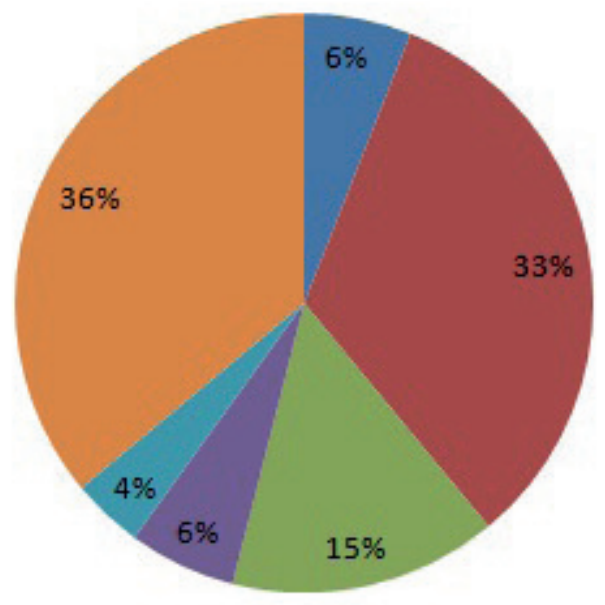

\author{
- Hijos de militares \\ - Hijos de refugiados \\ niños abandonados \\ Maternidad del Hospital \\ nanicomio Provincial \\ Desconocido
}

Fuente: elaboración propia a partir del libro Filiación de los huérfanos. 1924-1939. A.D.P.V. 
Este supuesto escenario de una inclusa saturada y con las adopciones restringidas también es plausible si tenemos en cuenta la legislación de la época. Durante los años treinta del siglo pasado se consolidaron tendencias reformistas y favorecedoras de la adopción que consideraban que el hijo adoptivo debía ser totalmente equiparado al hijo legítimo nacido en el seno del matrimonio. De ahí que para su adopción tenía que producirse la ruptura de vínculos entre el niño y su familia biológica. En este contexto, el Gobierno republicano trató de favorecer la adopción con el Decreto de 10 de abril de 1937, que pretendía, entre otros objetivos, flexibilizar las disposiciones del Código Civil en esta materia suprimiendo requisitos «para la adopción de quienes hubieran quedado huérfanos o desamparados a consecuencia de la guerra civil $»^{31}$. Sin embargo, los expertos han señalado que el decreto no consiguió sus objetivos, sobre todo la supresión de los vínculos entre el adoptado y su familia biológica (Calzadilla, 2003, pp. 28-29; Rodríguez Ennes, 2009, p. 132). Lo acontecido en la Inclusa de Valencia puede enmarcarse dentro del fracaso del Decreto de 10 de abril de 1937; es decir, la negativa de los responsables a entregar en adopción a niños que podían mantener vínculos con sus familias biológicas, acaso animados por una pronta resolución del conflicto bélico que favorecería la reagrupación familiar. La documentación consultada en el A.D.P.V. confirma el bloqueo de las adopciones: los expósitos entregados a familias para su lactancia decrecieron bruscamente en $1937(\mathrm{~N}=2)$ y $1938(\mathrm{~N}=2)$ con respecto a $1936(\mathrm{~N}=40)$ y $1939(\mathrm{~N}=23)^{32}$.

En la gráfica 2 también destaca el bajo porcentaje de niños procedentes de la maternidad del propio Hospital Provincial (6\%). Efectivamente, durante los años centrales del conflicto el número de niños acogidos en la Inclusa procedentes de la maternidad sufrió un descenso brusco $(1936 \mathrm{~N}=21 ; 1937 \mathrm{~N}=9 ; 1938 \mathrm{~N}=1$; $1939 \mathrm{~N}=30)^{33}$. Este contraste puede deberse a que durante la Guerra Civil los ingresos en la Inclusa de niños procedentes de la maternidad estaban muy controlados y debían estar plenamente justificados. Es lo que parecen indicar varios casos de niños cuyo ingreso en la Inclusa fueron aprobados porque «son hijos de una trabajadora del propio Hospital», o el caso de un «niño que ingresó en la Inclusa porque la madre aprovechando un momento en que no la observaban lo abandonó en la misma» ${ }^{34}$.

Finalmente, en la gráfica 3 hemos reflejado el organismo que ordenó el ingreso del niño en la Inclusa. Resulta llamativo que las órdenes de ingreso procedentes de la Dirección del Hospital constituyan un porcentaje discreto (19\%), sobre todo si tenemos en cuenta que éste era el mecanismo legal de ingreso más frecuente

Gráfica 3. Institución que ordenó el ingreso en la Inclusa (1936-1939)

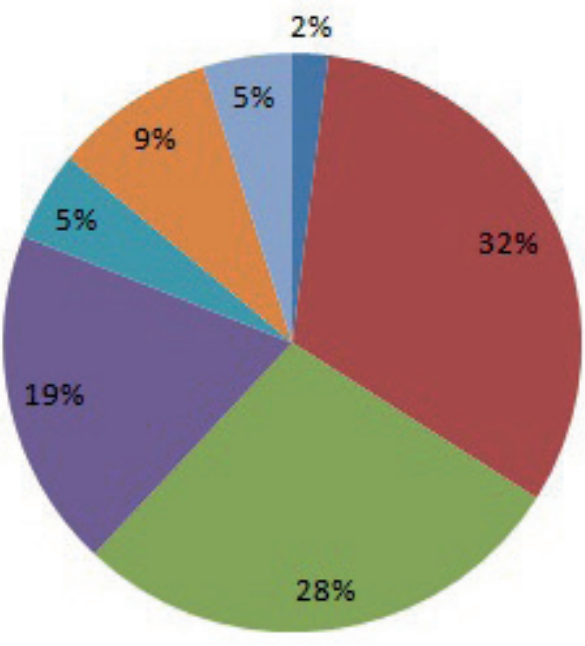

\author{
n Juzgado \\ - Consejo Provincial \\ Ministerio \\ Dirección Hospital \\ Dirección Manicomio \\ - Ingreso "provisional" \\ no consta
}

Fuente: elaboración propia a partir de datos consultados en Filiación de los huérfanos. 1924-1939. A.D.P.V. 
antes de la guerra; sin embargo, durante el conflicto las órdenes de ingreso en la Inclusa emanaron en un $62 \%$ de los casos del juzgado, del Consejo Provincial de Valencia, del Ministerio de Sanidad y Asistencia Social y del Consejo Nacional de Tutela de menores del Ministerio de Justicia. Se trataba en definitiva de presiones procedentes de diferentes organismos locales y estatales para el ingreso de niños en la inclusa. La documentación consultada, así pues, parece indicar que la Guerra Civil española alteró los criterios de ingreso en la Inclusa del Hospital Provincial de Valencia, prevaleciendo los de índole político-judicial sobre las decisiones técnicas.

\section{CONSIDERACIONES FINALES}

El lugar geoestratégico que ocupó la ciudad de Valencia durante la Guerra de España alteró profundamente el funcionamiento y la demografía sanitaria de la Inclusa del Hospital Provincial. Así, se establecieron criterios restrictivos de ingreso para la población infantil autóctona al objeto de proteger a la infancia que había quedado desamparada como consecuencia directa de la guerra. El primer colectivo perteneciente a esta infancia desvalida fue el de los niños refugiados, cuyo paradigma fueron los niños de la Inclusa de Madrid, que ocuparon la mayor parte de las instalaciones; el segundo fue el de los hijos de los militares que operaban en el frente de Levante, en cuyo caso la Inclusa se convirtió en un centro de acogida provisional mientras el padre combatía y la madre luchaba por sobrevivir. Un tercer colectivo lo constituyeron los hijos de refugiados cuyo paradero era desconocido. Por tanto, todo parece indicar que la Inclusa del Hospital Provincial de Valencia durante la Guerra Civil perdió su función original y actuó como un auténtico refugio para estos tres colectivos de población infantil.

El equilibrio de ingresos y de salidas de menores en la Inclusa valenciana se alteró drásticamente con motivo de la guerra. El cobijo ofrecido a los niños refugiados, junto con las dificultades legales para la adopción en aquellas circunstancias, se tradujo en un hacinamiento de la Inclusa valenciana y, a su vez, en una degradación progresiva de la asistencia sanitaria que allí se dispensaba. Lo que ocurrió una vez acabada la guerra podría ser reflejo del cambio drástico que experimentó la Inclusa de Madrid bajo las autoridades del nuevo Régimen (Jiménez López, 2013, pp. 369-386).

En definitiva, el caso de la Inclusa de Valencia resulta paradigmático de las limitaciones de la asistencia médica y social dispensada a la infancia refugiada por las instituciones valencianas, que se vieron absolutamente desbordadas por las dimensiones de la tragedia.

\section{NOTAS}

1. Este trabajo se enmarca en el proyecto del Ministerio de Economía y Competitividad (MINECO) "Hacia la consolidación de las especialidades médicas en Barcelona (19111939): unidad y división en el saber y la práctica de la medicina" (HAR2012-34586), y en parte se ha financiado gracias al presupuesto correspondiente a su tercera anualidad.

2. Durante la guerra el Asilo de San Rafael fue evacuado y transformado en prisión de mujeres y en hospital militar. Cf. Morales, 1960, p. 517.

3. La documentación sobre estas instalaciones sanitarias permanece en el Archivo de la Diputación Provincial de Valencia, inédita en gran parte.

4. Solamente los cuáqueros británicos atendieron en Cataluña a casi 150.000 niños. Cf. Serra, 2006, p. 199.

5. Correspondencia Consejería de Sanidad. 25 junio 1938. D.6.1 caja 1. Archivo de la Diputación Provincial de Valencia (A.D.P.V.)

6. Oficio del presidente del Comité Nacional de Refugiados al delegado de Asistencia Social. 6 diciembre 1936. D.6.1 c. 20. A.D.P.V.

7. Evacuación de niños a Girona. 8 enero 1936. D.6.1 c. 20. A.D.P.V.

8. Para obtener información sobre aspectos sanitarios de esta campaña, consúltese García Ferrandis; Munayco, 2010, pp. 245-249.

9. Colonia de Bétera. 25 diciembre 1936. D.6.1 c. 20. A.D.P.V.

10. Oficio del presidente del Comité Nacional de Refugiados al delegado de Asistencia Social. 11 enero 1937. D.6.1 c. 20. A.D.P.V.

11. Colonia de Buñol. 19 enero 1937. D.6.1 c. 20. A.D.P. V.

12. Informe de los servicios médicos del Hospital. Servicio de Enfermedades de la Infancia. 3 agosto 1936. I-2.4 c. 14 legajo 53. A.D.P.V.

13. Denuncia de Comín al Departamento de Hospitales. 30 abril 1937. D.6.1 c. 27, Hospital Provincial. A.D.P.V.

14. El Comité de Navajas ofrece al Comité Sanitario Popular unas casas para instalar un hospital. 18 septiembre 1936. D.6.1 c. 24, Hospital de Navajas. A.D.P.V. 
15. Denuncia de Comín al Departamento de Hospitales. 30 abril 1937. D.6.1 c. 27, Hospital Provincial. A.D.P.V. La palabra "destete" hace referencia a un niño mayor de 2 años, edad a la que finalizaba la lactancia en la inclusa.

16. Oficio del Departamento de Hospitales a la Inclusa de Madrid en Navajas. 5 mayo 1937. D.6.1 c. 27, Hospital Provincial. A.D.P.V.

17. Oficio del Departamento de Hospitales al Ministerio de Sanidad. [1937]. D.6.1 c. 15. A.D.P.V.

18. Partes diarios de ingresos de niños afectados por enfermedades infecciosas. Diciembre 1937-diciembre 1938 I-2.4 c. 8 legajo 36. A.D.P.V.

19. Oficio del Director del Hospital al Comité Sanitario. 25 diciembre 1936. D.6.1 c. 27, Hospital Provincial. A.D.P.V.

20. Oficio del Consejero de Sanidad al Director del Hospital. 16 junio 1937. D.6.1 c. 27, Hospital Provincial. A.D.P.V.

21. Expedientes de admisión de expósitos. 25 febrero 1937. II-4.1 c. 7 legajo 65. A.D.P.V.

22. Traslado desde la inclusa a la Casa de Misericordia. 7 junio 1937. II-4.4 c. 3 legajo 72. A.D.P.V.

23. Partes de defunción de niños evacuados de la Inclusa de Madrid e ingresados en el Hospital. Diciembre de 1936. II-2.4 c. 8 legajo 35. A.D.P.V.

\section{BIBLIOGRAFÍA}

Abreu, Laurinda (2003), "The Evora foundlings between the 16 th and the 19th century: the Portuguese public welfare system under analysis", Dynamis, 23, pp. 37-60.

Alonso Carballés, Jesús Javier (1998), 1937. Los niños vascos evacuados a Francia y Bélgica. Historia y memoria de un éxodo infantil, 1936-1940, Bilbao, Asociación de Niños Evacuados el 37.

Alted, Alicia (1996), "Las consecuencias de la Guerra Civil española en los niños de la República: de la dispersión al exilio", Espacio, Tiempo y Forma, Serie V, Historia Contemporánea, 9, pp. 207-228.

Alted, Alicia (2003), "Los niños de la Guerra Civil”, Anales de Historia Contemporánea, 9, pp. 43-58.

Anónimo (1937), "Rapport sur la mission sanitaire en Espagne (28 décembre 1936-15 janvier 1937)", Consultado en Revista de Sanidad e Higiene Pública, 5, pp. 297-332.

Anónimo (1939), "Food in Republican Spain", The British Medical Journal, 11 febrero, pp. 278-279.
24. Correspondencia de expósitos. 10 abril 1937. II-5.3 c. 15 legajo 113. A.D.P.V

25. Correspondencia de expósitos. 25 octubre 1937. II-5.3 c.15, legajo113. A.D.P.V.

26. Denuncia de Comín al Departamento de Hospitales. 14 septiembre 1937. D.6.1 c. 27, Hospital Provincial, A.D.P.V.

27. Oficios del Consejo Nacional de Tutela de Menores. Expedientes de admisión de expósitos. 1922-1950. II-4.1 c. 7 legajo 65. A.D.P.V

28. Filiación huérfanos. 1924-1939. II-7/6. A.D.P.V.

29. Expedientes de admisión de expósitos. 1922-1950. II-4.1 c. 7 legajo 65. A.D.P.V.

30. Signaturas 4354, 4360, 4363, 4364, 4366 y 4370. Archivo de la Diputación de Madrid. Tomado de Espina, 2005, p. 517.

31. Decreto de 10 de abril de 1937 sobre adopciones, Gaceta de la República de 13 de abril de 1937, no. 103, p. 179-180.

32. Libro de expósitos dados a lactar. 1909-1950. II-5/14. A.D.P.V; Criatures donades a lactar. 1936-1939. II-2.4 c. 82. A.D.P.V.

33. Libro del Torno. 1910-1940. II-3/32. A.D.P.V.

34. Filiación huérfanos. 1924-1939. II-7/6. A.D.P.V

Ballester, Rosa; Balaguer, Emilio (1995), "La infancia como valor y como problema en las luchas sanitarias de principios de siglo en España”, Dynamis, 15, pp. 177-192.

Barona, Carmen (2002), Organización sanitaria y de la higiene pública en la provincia de Valencia (1854-1936), Tesis doctoral, Universitat de València.

Barona, Josep Lluís (2002), Salud, enfermedad y muerte. La sociedad valenciana entre 1833 y 1939, Valencia, Institució Alfons el Magnànim.

Barona, Josep Lluís; Bernabeu, Josep (2007), La salud y el Estado. El movimiento sanitario internacional y la Administración española (1851-1945), Valencia, Publicacions de la Universitat de València.

Calzadilla, María Aranzazu (2003), “Las reformas del Código Civil español y la institución de la adopción", Anales de la Facultad de Derecho, 20, pp. 27-44.

Casademont, Adrià (2014), Les colònies infantils a Catalunya durant la Guerra Civil (1936-1939), Trabajo de Fin de Grado, Universitat de Girona. 
Clavijo, Julio (2003), La política sobre la població refugiada durant la Guerra Civil 1936-1939, Tesis doctoral, Universitat de Girona.

Crego Navarro, Rosalía (1989), "La colonias escolares durante la Guerra Civil (1936-1939)", Espacio,Tiempo y Forma, Serie V, Historia Contemporánea, 2, pp. 299-328.

del Cura, Ma Isabel; Huertas, Rafael (2007), Alimentación y enfermedad en tiempos de hambre. España, 1937-1947, Madrid, Consejo Superior de Investigaciones Científicas.

Eloy, Sarah (2005), De Spaanse migratie naar België gedurende de 19de en 20ste eeuw. "Los Niños de la Guerra»: Een casus, Gante, Universiteit Gent.

Escrivà, Cristina; Maestre, Rafael (2011), De las negras bombas a las doradas naranjas. Colonias escolares 1936-1939, Valencia, L'Eixam Edicions.

Espina, Pedro (2005), Historia de la Inclusa de Madrid, Madrid, Defensor del Menor en la Comunidad de Madrid.

Fernández, Juan Manuel (1987), "La asistencia a la infancia en la Guerra Civil. Las colonias escolares", Revista de Historia de la Educación, 6, pp. 83-128.

García Ferrandis, Xavier (2011), “El Hospital Provincial de Valencia durante la Guerra Civil española (1936-1939)". En: Hinojosa, José (coord.), El Hospital General de Valencia (1512-2012), cinco siglos de vanguardia sanitaria, Valencia, Fundació Hospital Reial i General, pp. 364-381.

García Ferrandis, Xavier (2015), L'assistència sanitària a la ciutat de Valencia durant la Guerra Civil, Valencia, Publicacions de la Universitat de València.

García Ferrandis, Xavier; Munayco, Armando José (2010), "La asistencia sanitaria en el frente de Teruel durante la primera campaña republicana (agosto de 1936-febrero de 1937)", Sanidad Militar, 66 (4), pp. 245-249.

García Sánchez, María Isabel (1999), “Mil cosas: la inclusa de Madrid", Historia 16, 275, pp. 92-97.

Jiménez López, Antonio (2013), “Vida y muerte en la Inclusa de Madrid en el primer año de posguerra", Espacio, Tiempo y Forma, Serie V, Historia Contemporánea, 25, pp. 369-386.

Kertzer, David (1999), "Syphilis, foundlings, and wetnurses in nineteenth-century Italy", Journal of Social History, 32 (3), pp. 589-603.

Labajos, Emilia; Vitoria, Fernando (1997), Los niños españoles refugiados en Bélgica (1936-1939), Namur, Asociación de los niños de la guerra.

Levene, Alysa (2005), "The estimation of mortality at the London Foundling Hospital, 1741-99", Population studies, 59 (1), pp. 87-97.
Lloret, Joan (2015), "La salud de la infancia: de la inclusa a la asistencia pediátrica". En: Barona, Josep Lluís; GuiIlem, Ximo (eds.), Sanidad internacional y transferencia del conocimiento científico. Europa, 1900-1975, Valencia, Publicacions de la Universitat de València.

McClure, Ruth (1981), Coram's children: the London Foundling Hospital in the eighteenth century, New Haven, Yale University Press.

Morales, Juan Luis (1960), El niño en la cultura española, Madrid, Imprenta T.P.A, p. 517.

Morell, Joan Ramon (2011), Solidaritat a Oliva 1936-1939, Gandia, CEIC Alfons el Vell.

Nicolson, Benedict and Kerslake, John (1972), The treasures of the Foundling Hospital, Oxford, Clarendon Press.

Ortega, José Antonio; Silvestre, Javier (2006), "Las consecuencias demográficas". En: Martín, Pablo; Martínez, Elena (eds.), La economía de la guerra civil, Madrid, Marcial Pons Historia.

Palacio, Irene (2003), Mujeres ignorantes: madres culpables. Adoctrinamiento y divulgación materno-infantil en la primera mitad del siglo XX, Valencia, Universitat de València.

Perdiguero, Enrique (comp.) (2004), Salvad al niño. Estudios sobre la protección a la infancia en la Europa mediterránea a comienzos del siglo XX, Valencia, Seminaris d'Estudis sobre la Ciència.

Perdiguero, Enrique; Castejón, Ramón (2009), “Auxilio social: Health care and social policies in Spain during and after de Civil War (1936-1939)". En: Andresen, Astri et al. (eds.), Healthcare Systems and Medical Institutions, Oslo, Novus Press, pp. 129-141.

Pérez Moreda, Vicente (2005), La infancia abandonada en España (siglos XVI-XX), Madrid, Real Academia de la Historia.

Presciutti, Diana (2011), “Dead infants, cruel mothers, and heroic popes: the visual rhetoric of foundling care at the hospital of Santo Spirito, Rome", Renaissance Society of America, 64 (3), pp. 752-799.

Ramírez, Susana María (2002), "Proyección científica de las ideas de Tomás Romay sobre la vacuna de la viruela en la Inclusa madrileña", Asclepio, 54 (2), pp. 109-128.

Revuelta, Bárbara Ana (2012), "Releasing mother's burdens: child abandonment and retrieval in Madrid, 1890-1935", Journal of Interdisciplinary History, 42 (4), pp. 645-672.

Revuelta, Bárbara Ana (2015), “¿Qué pasa en La Inclusa? The role of press scandals, doctors and public authorities in the evolution of La Inclusa de Madrid, 1890-1935", Dynamis, 35 (1), pp. 107-130.

Rodríguez Ennes, Luis (2009), “La adopción romana: continuidad y discontinuidad de un modelo", Dereito, 18 (1), pp. 115-134. 
Rodríguez Ocaña, Esteban (2003), “La salud infantil, asunto ejemplar en la historiografía contemporánea", Dynamis, 23, pp. 27-36.

Russell, Audrey (1938), “Hunger in Spain”, Lancet, 6018, p. 1419.

Sagués, Joan (2001), "La historia de la Guerra Civil española, un campo con puertas aún por abrir", Ayer, 43, pp. 277-289.

Selfa, José (1953), Reumatismo y corea en la provincia de Valencia, Madrid, Dirección General de Sanidad, pp. 32-34.

Sheetz-Nguyen, Jessica (2012), Victorian women, unwed mothers and the London Foundling Hospital. London, New York, Continuum.

Serra, Rosa (2006), L'ajuda humanitària dels quàquers als infants de Catalunya durant la Guerra Civil, 1936/1939, Tesis doctoral, Universitat de Girona.
Vidal, Florentina; Vidal, Benicia (1995), Bordes y bastardos. Una historia de la Inclusa de Madrid, Madrid, Compañía Literaria.

Vinyes, Ricard (2002), Los niños perdidos del franquismo, BarceIona, Plaza y Janés.

Zafra, Enrique; Crego, Rosalía; Heredia, Carmen (1997), Los niños españoles evacuados a la URSS (1937), Namur, Asociación de los niños de la guerra.

Zafra, Miguel; Arana, José Ignacio de (2015), "Enrique Jaso y su epopeya en el traslado de los niños de la Inclusa de Madrid", Cuadernos de Historia de la Pediatría Española, 10, pp. 8-16. 